\title{
Cavity-enhanced absorption and Fano resonances in graphene nanoribbons
}

\author{
Ivan V. Iorsh, ${ }^{1}$ Ilya V. Shadrivov, ${ }^{2}$ Pavel A. Belov, ${ }^{1}$ and Yuri S. Kivshar ${ }^{1,2}$ \\ ${ }^{1}$ National Research University of Information Technologies, Mechanics and Optics (ITMO), St. Petersburg 197101, Russia \\ ${ }^{2}$ Nonlinear Physics Center, Research School of Physics and Engineering, Australian National University, Canberra, \\ Australian Capital Territory 0200, Australia
}

(Received 2 September 2013; published 20 November 2013)

\begin{abstract}
We analyze the absorption of a graphene nanoribbon placed into an optical cavity. We demonstrate the existence of strong coupling between the Van-Hove singularities in armchair graphene nanoribbons and the modes of optical cavity, and calculate the dispersion of the resulting hybrid eigenmodes of the structure. We demonstrate that the total absorption of the structure can be enhanced dramatically being tuned via the Fano resonance.
\end{abstract}

DOI: 10.1103/PhysRevB.88.195422

PACS number(s): 78.67.Wj, 42.60.Da

\section{INTRODUCTION}

Graphene is a two-dimensional lattice of carbon atoms that exhibits a wide range of unique electronic properties such as linear dispersion of charge carriers, Berry phase, and roomtemperature quantum Hall effect. ${ }^{1-3}$ Beyond pure electronic properties, graphene attracts significant research interest due to its electromagnetic properties since the graphene layer can be described essentially as a two-dimensional current which can support highly localized surface electromagnetic waves - surface plasmon polaritons. Surface plasmons excited at individual graphene sheets have been extensively studied both theoretically ${ }^{4-10}$ and experimentally. ${ }^{11,12}$ Graphene surface plasmons are excited in the $\mathrm{THz}$ frequency range which is extremely important for biomedical and security applications, ${ }^{13}$ therefore the field of graphene plasmonics ${ }^{14}$ is rapidly growing. While graphene plasmonics is also very promising for optoelectronic applications, it inherited a number of serious restrictions from conventional plasmonics. These are the inevitable Joule losses and other damping mechanisms ${ }^{15}$ leading to smaller plasmon propagation lengths and associated difficulties with the excitation of surface plasmon polaritons. Moreover, a single-atom thickness of graphene restricts its ability to absorb or emit light, limiting the performance of prospective optoelectronic devices.

A prominent approach for controlling graphene properties is based on the use of narrow graphene stripes, or nanoribbons. One of the useful properties of the graphene nanoribbon, as compared to planar graphene, is its ability to behave as a semiconductor whose band gap can be tuned in a wide frequency range without any additional strain or an external potential. Additionally, it has been shown experimentally that a graphene layer placed in a planar cavity can significantly increase the light-matter interaction and the performance of the graphene-based transistors ${ }^{16,17}$ and photodetectors. ${ }^{18,19}$ As was shown for one-dimensional semiconductors placed in a resonator, a strong coupling may occur between interband transitions and optical cavity modes. ${ }^{20}$ Here we employ this idea for graphene and study the properties of hybrid eigenmodes of a narrow graphene nanoribbon placed into an optical cavity. In this article we suggest a novel approach for graphene optoelectronics based on the physics of graphene nanoribbons in an optical cavity. We show that this system supports hybrid modes in the THz and far-IR frequency ranges which can be excited electrically. We observe that the interaction of cavity modes with interband transitions results in the characteristic Fano-like shape of the absorption spectrum giving rise to a way for controllable and enhanced absorption of graphene.

\section{HYBRID STRUCTURE AND INTERACTIONS}

We consider the structure shown schematically in Fig. 1(a); it contains a graphene nanoribbon placed in an optical cavity. The energy spectrum and wave functions of electrons in the nanoribbon were studied previously by the authors of Ref. 21, and the band diagram of the armchair nanoribbon of the width of $9 \mathrm{~nm}$ is shown in Fig. 1(b). In what follows, we consider only the armchair nanoribbon since for zigzag nanoribbons the depolarization effects are much stronger and the absorption peaks are wider, and thus strong coupling effects are suppressed.

Due to a finite width of the nanoribbon, the wave-vector component perpendicular to the axis of nanoribbon is quantized, and it can be written as

$$
k_{n}=\frac{2 \pi}{3 a_{0}}\left(1+\frac{3 n}{2\left(W / a_{0}\right)+1}\right),
$$

where $a_{0}$ is the interatomic spacing, $W$ is the nanoribbon width, and $n$ is an integer. If $W=(3 M+1) a_{0}$, where $M$ is an integer, there appears a subband where $k_{n}$ vanishes; the dispersion equation of this subband is given by

$$
\varepsilon_{n}=s \hbar v_{F} \sqrt{k_{n}^{2}+k_{y}^{2}}=s \gamma a_{0} \sqrt{k_{n}^{2}+k_{y}^{2}} .
$$

In Eq. (2), $s$ is the band index which is $s=+1$ for electrons and $s=-1$ for holes, $k_{y}$ is the electron wave vector along the nanoribbon, and $\gamma$ is the hopping energy for the electrons which is approximately equal to $3 \mathrm{eV}$. Below we consider the nanoribbon with the width $W=(3 M+2) a_{0}$ since we are interested in the absorption peak at the band edge.

The band structure is characterized by the gap $E_{G}$ [see Fig. 1(b)],

$$
E_{G}=\gamma \frac{8 \pi}{(18 M+3)} .
$$

We choose $M=20$ which corresponds to the nanoribbon of the width $W=62 a_{0}=9 \mathrm{~nm}$. For this nanoribbon, the band gap is $0.2 \mathrm{eV}$ which is approximately $50 \mathrm{THz}$. The Hamiltonian 


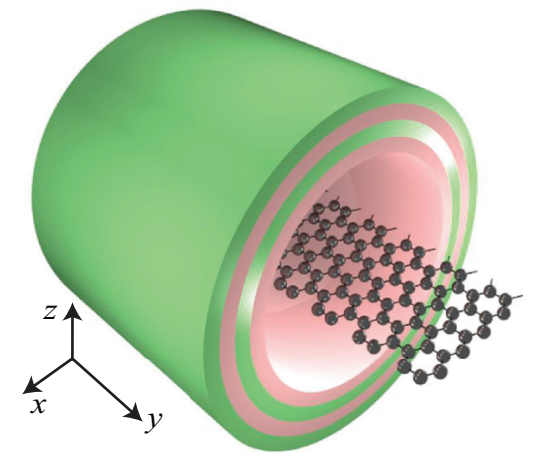

(a)

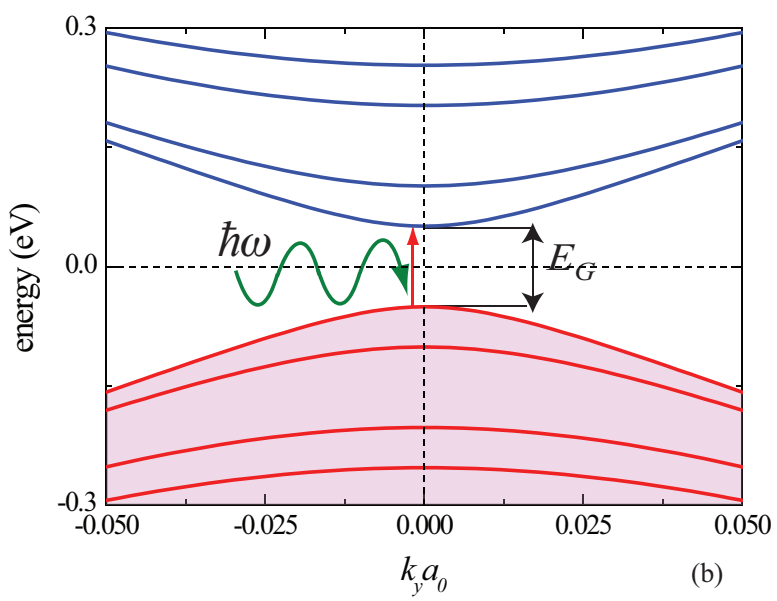

FIG. 1. (Color online) (a) Schematic of the structure: An armchair graphene nanoribbon is placed in an optical cavity. (b) Band diagram of the armchair nanoribbon.

of the nanoribbon is given by a $(4 \times 4)$ matrix

$H=\gamma_{0} a_{0}\left(\begin{array}{cccc}0 & k_{n}-i k_{y} & 0 & 0 \\ k_{n}+i k_{y} & 0 & 0 & 0 \\ 0 & 0 & 0 & -k_{n}-i k_{y} \\ 0 & 0 & -k_{n}+i k_{y} & 0\end{array}\right)$.

Next, we introduce a bare interband polarization of a nanoribbon. We account only for the vertical transitions since the light wave vector is much less than the electron wave vectors. Polarization is calculated via the Fourier transformation of the Green's functions, and a summation runs over the Matsubara frequencies. ${ }^{22,23}$ The final expression for the polarization can be written as

$$
\begin{aligned}
& \Pi_{0}(\omega) \\
& =L_{y} \int d k_{y}\left[f_{h}\left(k_{y}\right)-f_{e}\left(k_{y}\right)\right] \\
& \quad \times\left\{\frac{1}{\hbar \omega-2 \varepsilon_{1}\left(k_{y}\right)+i \hbar / \tau}-\frac{1}{\hbar \omega+2 \varepsilon_{1}\left(k_{y}\right)-i \hbar / \tau}\right\},
\end{aligned}
$$

where $f_{h}$, and $f_{e}$ are the Fermi distribution functions, $\varepsilon_{1}$ is the energy of the first subband, $\tau$ is the electron scattering time (which we set below to $1 \mathrm{ps}$ ), and $L_{y}$ is the length of the nanoribbon. Absorption is proportional to the imaginary part of the polarization. Due to the one-dimensional nature of the effective geometry, the density of states has a peak
$1 / \sqrt{E-E_{G}}$ close to the band edge that corresponds to a Van-Hove singularity. ${ }^{20}$

For a nanoribbon placed in an optical cavity, the mode coupling becomes strong leading to a substantial absorption enhancement. Following the authors of Refs. 20 and 23, we introduce the propagator of the cavity photon in the following form:

$$
D_{\mathrm{ph}}=\frac{2 \hbar \omega_{\mathrm{cav}}(q)}{\hbar^{2} \omega^{2}-\hbar^{2} \omega_{\mathrm{cav}}^{2}(q)+2 i \gamma_{\mathrm{cav}} \hbar \omega_{\mathrm{cav}}(q)},
$$

where $\omega_{\text {cav }}(q)$ is the dispersion of the cavity mode and $\gamma_{\text {cav }}$ is the damping of the cavity mode. The full polarization of the system then can be presented in the following form: ${ }^{23}$

$$
\Pi=\frac{\Pi_{0}}{\left(1-g^{2} D_{\mathrm{ph}} \Pi_{0}\right)} .
$$

Here $g$ is the light-matter coupling constant which is equal to the dipole transition matrix element, and the electric field is normalized to the energy of one photon. The dipole matrix elements have been calculated by the authors of Refs. 21 and 24 , and for our case the dipole matrix element is given by

$$
g=e v_{F} A_{0},
$$

where $A_{0}$ is derived from the normalization condition

$$
\frac{1}{2} \varepsilon_{0} E_{0}^{2} \pi R^{2} L_{y}=\frac{1}{2} \varepsilon_{0} \omega_{\text {cav }}^{2} A_{0}^{2} \pi R^{2} L_{y}=\hbar \omega_{\text {cav }},
$$

where $R$ is an effective radius of the optical cavity. We use such a simplified form of the dipole matrix element (7) since the main contribution to the polarization (4) comes from the electron transitions close to the band gap and thus small in-plane momenta. Expression (7) is obtained as a limiting case for the value of the matrix element for vanishing in-plane momenta. Here we consider the cavity eigenmode which has the component of the electric field parallel to the ribbon since the interband absorption threshold frequency for the perpendicular polarization is slightly blue-shifted $\left(E_{g} \approx\right.$ $0.3 \mathrm{eV}$ ) due to different selection rules, ${ }^{24}$ and we can neglect the intraband absorption if the Fermi energy lies in the band gap.

The condition for a strong coupling can be simplified to the following:

$$
g>\left|\gamma_{\text {cav }}-\hbar / \tau\right|,
$$

which can be easily achieved for realistic nanoribbons with $g$ of the order of $1 \mathrm{meV}$, and the difference of $\gamma_{\text {cav }}$ and $\hbar / \tau$ can be made arbitraryily small.

Finally, we study the conventional absorption in our hybrid cavity-nanoribbon system. To calculate the absorption, we multiply our result for the polarization by the dimensional constant $\chi$ given by

$$
\chi=\frac{e^{2} v_{F}^{2}}{\varepsilon_{0} \pi R^{2} \omega_{\mathrm{cav}}^{2}}\left(\frac{\omega}{c}\right) .
$$

The absorption map is shown in Fig. 2(a). In the modeling, we consider a cylindrical cavity with the ground state energy of $0.18 \mathrm{eV}$ which corresponds to $R \approx 2.5 \mu \mathrm{m}$. We observe that for small wave vectors two absorption peaks appear: a low-energy narrow peak [see Fig. 2(b)] corresponding to a cavity mode and a wide-peak corresponding to the Van-Hove singularity in the nanoribbon. We then observe the anticrossing 

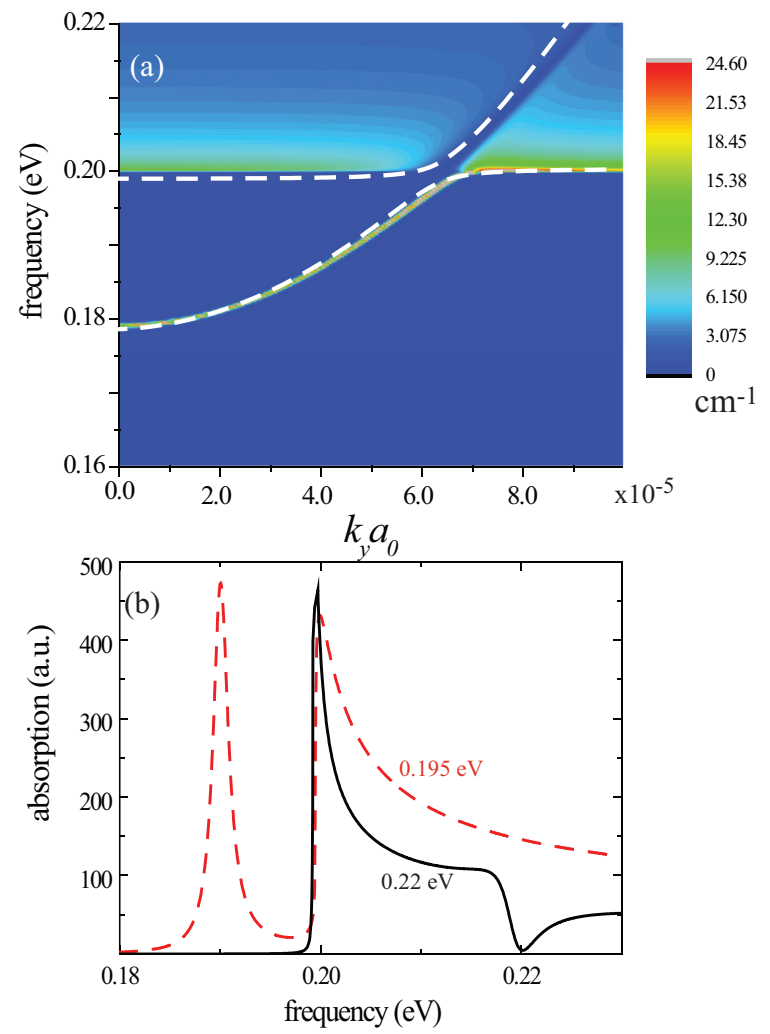

FIG. 2. (Color online) (a) Absorption map of the graphene nanoribbon in a cavity. Dispersion of the eigenmodes is shown by white dashed curves. (b) Absorption spectra for the cases when the cavity frequency is tuned to 0.22 (solid line) and $0.195 \mathrm{eV}$ (dashed line).

behavior between these two resonances at $k_{y} a_{0} \approx 7 \times 10^{-5}$. After the anticrossing point, one of the modes still corresponds to the absorption peak, whereas the other mode yields a transmission maximum with a relatively narrow dip in the absorption. The dispersion of the eigenmodes of the system that correspond to the zeros of the denominator in Eq. (6) is shown with white dashed lines in Fig. 2(a).

\section{FANO RESONANCE}

A dip in the absorption spectrum observed for the cavity mode frequencies equal to or larger than the energy band gap resembles the shape of the Fano resonance. ${ }^{25}$ In our problem, the Fano resonance originates from an interference between a wide interband polarization and a narrow cavity mode resonance. To verify our assumption, below we derive an analytical expression for the total absorption in the hybrid cavity-nanoribbon system and compare it to the conventional Fano formula.

To derive the Fano formula for the total absorption, first we assume that the cavity-mode frequency is positively detuned from the band gap, and thus the interband polarization is effectively frequency independent in the vicinity of the cavity-mode resonance. Thus, the interband polarization can be written as $\Pi_{0}=P e^{i \phi}$, where $P$ and $\phi$ are the polarization amplitude and phase, respectively. In the vicinity of the cavity resonance, the propagator of the cavity mode can be presented as

$$
D_{\mathrm{ph}} \approx \frac{1}{\gamma_{\mathrm{cav}}} \frac{1}{(\delta-i)},
$$

where $\delta=\left(\omega-\omega_{\text {cav }}\right) / \gamma_{\text {cav }}$ is a relative detuning from the resonance frequency. We then substitute the simplified interband and cavity polarizations into Eq. (6), and derive an analytical result for the total absorption $\alpha$,

$$
\alpha=\operatorname{Im} \Pi=P \frac{\mathcal{P}+\left(\delta^{2}+1\right) \sin \phi}{(\delta-\mathcal{P} \cos \phi)^{2}+(1+\mathcal{P} \sin \phi)^{2}},
$$

where $\mathcal{P}=g^{2} P / \gamma_{\text {cav }}$ denotes the strength of the coupled resonance. Taking the derivative of Eq. (12) over $\delta$, we find the extremum points of the total absorption $\delta_{1,2}$,

$$
\delta_{1}=-\cot \phi, \quad \delta_{2}=\tan \phi\left(1+\frac{\tilde{P}}{\sin \phi}\right),
$$

and the values of the total absorption at the extremum points $\alpha_{1,2}$ :

$$
\begin{gathered}
\alpha_{1}=\frac{\operatorname{Im} \Pi_{0}}{1+\tilde{P} \sin \phi}, \\
\alpha_{2}=\frac{\operatorname{Im} \Pi_{0}}{\sin ^{2} \phi}\left(1-\frac{\cos ^{2} \phi}{1+\tilde{P} \sin \phi}\right) .
\end{gathered}
$$

We observe that in the vicinity of the band-gap frequency the real part of the interband polarization is negative, and since the imaginary part is always positive, the condition $\cot \phi, \tan \phi<0$ is satisfied. This means that $\delta_{1}$ is blue-shifted from the cavity resonance and $\delta_{2}$ is red-shifted. Moreover, when we consider the noninteracting case with $g=0$, the values of absorption in both extremum points are identical and equal to $\operatorname{Im} \Pi_{0}$, as can be easily seen from Eqs. (14) and (15). However, when we take into account the coupling, and if the cavity is characterized by a high-quality factor such that $\mathcal{P} \gtrsim 1$, then the extremum point $\delta_{1}$ corresponds to a dip in absorption where the absorption is suppressed by $\tilde{P} \sin \phi$, and the extremum point $\delta_{2}$ corresponds to a peak in the absorption where it is increased by $1 / \sin ^{2} \phi$.

In Fig. 3, we compare the analytical model for absorption to the numerical results. The parameters are $\gamma_{x}=10 \mathrm{meV}, \gamma_{c}=$ $0.1 \mathrm{meV}, g=1 \mathrm{meV}$, and $\omega_{\text {cav }}=E_{G}=0.2 \mathrm{eV}$. To observe the predicted effects, we need a reasonably high-quality cavity with the quality factor around $10^{3}$. In this case, the analytical approximation exhibits a good agreement with the numerical results.

Finally, we would like to mention that several other manybody effects may occur in this hybrid cavity-nanoribbon structure, such as the interaction of the excitons of the nanoribbon with cavity modes. Excitonic properties of nanoribbons have been studied in several works. ${ }^{26,27}$ In particular, a simple relation between the exciton binding energy and the armchair nanoribbon width has been obtained in the form ${ }^{27}$

$$
E_{b} \approx 0.46 W^{-0.55},
$$

where $E_{b}$ is the exciton binding energy (in $\mathrm{eV}$ ) and $W$ is the ribbon width (in $\mathrm{nm}$ ). For the considered nanoribbon with the width of $9 \mathrm{~nm}$, the binding energy is equal to $0.12 \mathrm{eV}$, which corresponds to the ground exciton energy of $80 \mathrm{meV}$. Two points should be mentioned here. First, the binding energy is 


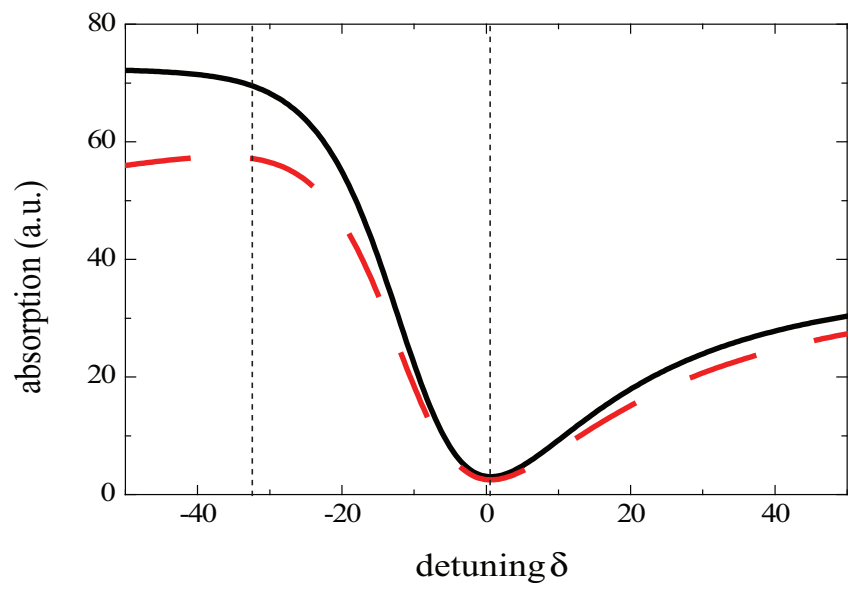

FIG. 3. (Color online) Comparison of the analytically obtained absorption to numerical results. Red dashed line corresponds to the analytical result obtained with Eq. (12), and black solid line corresponds to numerical simulations. Vertical dotted lines mark the positions of the analytically obtained extremum points defined by Eq. (13).

significantly larger than that of the excitons in conventional semiconductor quantum wells, and it is larger than the room temperature thermal energy of $26 \mathrm{meV}$; this means that the excitonic effects can be observed at room temperature. While the excitonic condensation effects are hardly expectable in this one-dimensional structure, this system could benefit from the fact that a ratio of the exciton frequency and the light-matter coupling parameter $g$ is much less than that in conventional semiconductor quantum wells. This means that the effects of ultrastrong coupling ${ }^{28}$ can be observed in our hybrid system, for which the conventional Jaynes-Cummings model is not applicable, and many new quantum electrodynamics effects may exist.

\section{CONCLUSION}

We have revealed the existence of strong light-matter interaction between photonic modes of the one-dimensional optical cavity and interband transitions in the graphene nanoribbon. We have described the anticrossing effect for the eigenmodes of the hybrid system characterized by a Fano-like resonance. We have noticed that the coupling of nanoribbon excitons to optical cavity modes can lead to a number of unconventional phenomena in the ultrastrong coupling regime.

\section{ACKNOWLEDGMENTS}

The work was supported by the Australian Research Council and the Ministry of Education and Science of the Russian Federation. The authors thank Yu. V. Bludov, N. M. R. Peres, and Ivan Shelykh for useful comments.
${ }^{1}$ R. R. Nair, P. Blake, A. N. Grigorenko, K. S. Novoselov, T. J. Booth, T. Stauber, N. M. R. Peres, and A. K. Geim, Science 320, 1308 (2008).

${ }^{2}$ F. Bonaccorso, Z. Sun, T. Hasan, and A. C. Ferrari, Nat. Photon. 4, 611 (2010).

${ }^{3}$ Q. Bao and K. P. Loh, ACS Nano 6, 3677 (2012).

${ }^{4}$ S. A. Mikhailov and K. Ziegler, Phys. Rev. Lett. 99, 016803 (2007).

${ }^{5}$ Yu. V. Bludov, M. I. Vasilevskiy, and N. M. R. Peres, Europhys. Lett. 92, 68001 (2010).

${ }^{6}$ F. H. L. Koppens, D. E. Chang, and F. J. G. de Abajo, Nano Lett. 11, 3370 (2011).

${ }^{7}$ M. Jablan, H. Buljan, and M. Soljacic, Opt. Exp. 19, 11236 (2011). ${ }^{8}$ A. Y. Nikitin, F. Guinea, F. J. Garcia-Vidal, and L. Martin-Moreno, Phys. Rev. B 84, 195446 (2011).

${ }^{9}$ A. Vakil and N. Engheta, Science 332, 1291 (2011).

${ }^{10}$ B. E. Sernelius, Phys. Rev. B 85, 195427 (2012).

${ }^{11}$ J. Chen, M. Badioli, P. Alonso-González, S. Thongrattanasiri, F. Huth, J. Osmond, M. Spasenović, A. Centeno, A. Pesquera, P. Godignon, A. Z. Elorza, N. Camara, F. J. García de Abajo, R. Hillenbrand, and F. H. L. Koppens, Nature (London) 487, 77 (2012).

${ }^{12}$ Z. Fei, A. S. Rodin, G. O. Andreev, W. Bao, A. S. McLeod, M. Wagner, L. M. Zhang, Z. Zhao, M. Thiemens, G. Dominguez, M. M. Fogler, A. H. Castro Neto, C. N. Lau, F. Keilmann, and D. N. Basov, Nature (London) 487, 82 (2012).

${ }^{13}$ J. M. Chamberlain, Philos. Trans. R. Soc. London A 362, 199 (2004).

${ }^{14}$ A. N. Grigorenko, M. Polini, and K. S. Novoselov, Nature Photonics 6, 749 (2012).
${ }^{15}$ H. Yan, T. Low, W. Zhu, Y. Wu, M. Freitag, X. Li, F. Guinea, P. Avouris, and F. Xia, Nat. Photon. 7, 394 (2013).

${ }^{16}$ M. Engel, M. Steiner, A. Lombardo, A. C. Ferrari, H. Lohneysen, P. Avouris, and R. Krupke, Nat. Commun. 3, 906 (2012).

${ }^{17}$ X. Gan, K. F. Mak, Yu. Gao, Yu. You, F. Hatami, J. Hone, T. F. Heinz, and D. Englund, Nano Lett. 12, 5626 (2012).

${ }^{18}$ M. Furchi, A. Urich, A. Pospischil, G. Lilley, K. Unterrainer, H. Detz, P. Klang, A. M. Andrews, W. Schrenk, G. Strasser, and T. Mueller, Nano Lett. 12, 2773 (2012).

${ }^{19}$ A. Ferreira, N. M. R. Peres, R. M. Ribeiro, and T. Stauber, Phys. Rev. B 85, 115438 (2012).

${ }^{20}$ K. B. Arnardottir, O. Kyriienko, M. E. Portnoi, and I. A. Shelykh, Phys. Rev. B 87, 125408 (2013).

${ }^{21}$ L. Brey and H. A. Fertig, Phys. Rev. B 75, 125434 (2007).

${ }^{22} \mathrm{H}$. Bruus and K. Felnsberg, Many-Body Quantum Theory in Condensed Matter Physics (Oxford University Press, New York, 2004).

${ }^{23}$ O. Kyriienko and I. A. Shelykh, J. Nanophoton. 6, 061804 (2012).

${ }^{24}$ K. I. Sasaki, K. Kato, Ya. Tokura, K. Oguri, and T. Sogawa, Phys. Rev. B 84, 085458 (2011).

${ }^{25}$ A. E. Miroshnichenko, S. Flach, and Yu. S. Kivshar, Rev. Mod. Phys. 82, 2257 (2010)

${ }^{26}$ L. Yang, M. L. Cohen, and S. G. Louie, Nano Lett. 7, 3112 (2007).

${ }^{27}$ X. Zhu and H. Su, J. Phys. Chem. A 115, 11998 (2011).

${ }^{28}$ T. Niemczyk, F. Deppe, H. Huebl, E. P. Menzel, F. Hocke, M. J. Schwarz, J. J. Garcia-Ripoll, D. Zueco, T. Hämmer, E. Solano, A. Mark, and R. Gross, Nat. Phys. 6, 772 (2010). 